\title{
FREQÜÊNCIA RESPIRATÓRIA E TEMPERATURA CLOACAL EM FRANGOS DE CORTE SUBMETIDOS À TEMPERATURA AMBIENTE CÍCLICA ELEVADA
}

(Respiratory frequency and cloacal temperature in broiler chickens submitted to high cyclic ambient temperature)

MARCHINI,C.F.P.'; SILVA, P.L. ${ }^{2}$; NASCIMENTO, M.R.B.M²; TAVARES, M $^{3}$.

${ }^{1}$ Universidade de Franca - SP.

${ }^{2} F A M E V$ - UFU.

${ }^{3}$ FAMAT - UFU.

\begin{abstract}
RESUMO - Este estudo objetivou verificar os efeitos da temperatura ambiente cíclica elevada sobre a freqüência respiratória e temperatura cloacal em frangos de corte. Setenta pintos da linhagem Avian, machos foram alojados em baterias de gaiolas de julho a agosto de 2004 e divididos em dois grupos. O primeiro foi submetido diariamente, durante uma hora, à temperatura ambiente elevada do primeiro até o $42^{\circ}$ dia de idade (grupo ST). O segundo mantido em temperatura de conforto térmico (grupo TN). Analisou-se semanalmente a freqüência respiratória e temperatura cloacal, de dez aves do grupo ST, antes e após o estresse por calor. No grupo TN, estes parâmetros foram avaliados entre 12 e 13 horas. O delineamento foi feito em parcela subdividida "Split-Plot" no tempo. O efeito temperatura ambiente foi considerado parcela, enquanto que o efeito idade, subparcela. Os dados foram analisados pela análise de variância e as médias comparadas pelo teste de Tukey a $5 \%$. As aves ST e TN apresentaram aumento da temperatura cloacal com a idade. No grupo TN, a freqüência respiratória reduziu com a idade. As aves do ST tiveram aumento na freqüência respiratória e temperatura cloacal depois do estresse pelo calor. Assim, a temperatura corporal aumenta com a idade do frango, independentemente da temperatura ambiente, e a freqüência respiratória reduz com a idade para as aves em termoneutralidade. Frangos de corte submetidos à temperatura ambiente cíclica elevada apresentam aumento na freqüência respiratória e na temperatura cloacal.
\end{abstract}

Palavras-chave: parâmetros fisiológicos; estresse térmico; aves; ambiência

ABSTRACT - The aim of this study was to verify the effect of high cyclic ambient temperature on the respiratory frequency and cloacal temperature in broiler chickens. Seventy male, Avian breed chickens were housed in batteries of cages from July to August 2004, and divided into two groups. The first group was submitted to a high ambient temperature for one hour, daily, from the first to the 42nd day of age (Group ST). The second was kept at a thermally confortable temperature (Group TN). Respiratory frequency and cloacal temperature were analyzed weekly in ten birds from the ST group, before and after heat stress. In Group TN, these parameters were assessed between the times of 12:00 and 13:00hrs. Delineation was done in Split-Plot in time. The effect of ambient temperature was considered the plot, while the effect of age was taken as the split-plot. The data were analyzed by the analysis of variance, and the means were compared by the Tukey test at $5 \%$. The birds in ST and TN displayed increase in cloacal temperature with age. In Group TN, the respiratory frequency diminished with age. The birds in ST had increased respiratory frequency and cloacal temperature after heat stress. Thus, the body temperature increases with the chicken's age, irrespective of the ambient temperature, and respiratory frequency decreases with age for birds at thermoneutrality. Chickens for slaughter submitted to high cyclic ambient temperature presented increase in respiratory frequency and cloacal temperature.

Key Words: physiologic parameters; heat stress; birds; ambiance.

\section{INTRODUÇÃO}

Em países tropicais como o Brasil, as condições de conforto térmico dificilmente são obtidas, visto que, durante quase o ano todo a temperatura ambiente, a intensidade de radiação solar e umidade do ar são muito elevadas. O frango de corte é muito sensível à temperatura ambiente elevada, tendo seu desempenho prejudicado resultando em crescimento retardado e baixo peso à 
MARCHINI, C.F.P. et al.

idade de abate além desta causar aumento na temperatura retal e na freqüência respiratória (SILVA et al., 2003) e estresse pelo calor (DIONELLO et al., 2002). Portanto, o ambiente no qual a ave é criada pode influenciar o seu desempenho (FURLAN et al.; 2001).

À medida que os efeitos da temperatura ambiente elevada sobre as aves se intensificam, diversas respostas fisiológicas são desencadeadas com o objetivo de aumentar a dissipação de calor por elas (ALTAN et al., 2003). A freqüência respiratória aumenta durante o estresse por calor (BORGES et al., 2003) para estimular a perda evaporativa e manter o equilíbrio térmico corporal, ou seja, resfriar melhor o corpo (FURLAN e MACARI, 2002). Este aumento constitui a principal e mais eficiente forma de dissipar calor em aves submetidas a altas temperaturas (OLIVEIRA NETO et al., 2000) e pode resultar em alcalose respiratória, provocando piora de desempenho zootécnico (BORGES et al., 2003).

O aumento da temperatura retal é uma resposta fisiológica às condições de temperatura $\mathrm{e}$ umidade elevadas que resulta do armazenamento do calor metabólico (SILVA et al., 2003). Ao avaliar lotes de frangos de corte, ALTAN et al. (2003) observaram que, com a elevação da temperatura ambiente, a temperatura corporal aumenta.

A maioria dos estudos sobre os efeitos do estresse por calor sobre a fisiologia das aves têm sido conduzida em temperaturas ambientes elevadas constantes, embora sob condições naturais não o seja. Dessa forma, este estudo objetivou verificar o efeito da temperatura ambiente cíclica elevada, por uma hora, do primeiro ao $42^{\circ}$ dia de idade sobre a freqüência respiratória e temperatura cloacal de frangos de corte machos.

\section{MATERIAL E MÉTODO}

Setenta pintos de corte da linhagem Avian, machos, foram alojados em baterias de quatro gaiolas, medindo $160 \times 50 \times 60 \mathrm{~cm}$ cada e colocadas sobre estrado de madeira a $50 \mathrm{~cm}$ do solo, em local aberto, ventilado e sombreado. As aves foram criadas em ambiente com temperaturas dentro da termoneutralidade, variando de $32^{\circ}$ a $35^{\circ} \mathrm{C}$ na primeira semana de idade depois da qual esta foi diminuída gradualmente a $21^{\circ} \mathrm{C}$ no $35^{\circ}$ até o $42^{\circ}$ dia de idade. A temperatura ambiente foi mantida por equipamentos convencionais de aquecimento e resfriamento. Água e ração comercial foram fornecidas à vontade e as aves foram submetidas a 24 horas de luz natural e artificial durante o período experimental, realizado de julho e agosto de 2004 , em Franca - SP. No primeiro dia de idade as aves foram divididas aleatoriamente em dois grupos com 35 aves cada. Em um, as aves foram mantidas em ambiente com temperatura dentro da zona de termoneutralidade (grupo TN) e no outro, foram submetidas à temperatura ambiente de $38^{\circ} \mathrm{C}$, de 12 a 13 horas, do primeiro ao $27^{\circ}$ dia de idade e à $40^{\circ} \mathrm{C}$ durante uma hora do $28^{\circ}$ ao $42^{\circ}$ dia de idade (grupo ST). Para aquecer o ambiente foram utilizadas quatro lâmpadas incandescentes de 200 watts em cada gaiola. Uma lona plástica transparente foi colocada sobre as gaiolas para que o calor não dissipasse para o ambiente. Durante a exposição das aves à temperatura ambiente cíclica elevada (TACE), esta foi monitorada com um termômetro com coluna de mercúrio com precisão de um grau centígrado colocado na altura do dorso das aves, e água e ração foram suprimidas. Terminada a exposição à $\mathrm{TACE}$, as aves recebiam água e ração à vontade e eram mantidas em temperatura de conforto térmico durante 23 horas.

A freqüência respiratória $(M R / m)$ e a temperatura cloacal (TC) de dez aves do grupo ST, antes e imediatamente após serem submetidas à TACE, foram obtidas no $1^{\circ}, 7^{\circ}, 14^{\circ}, 21^{\circ}, 28^{\circ}, 35^{\circ} \mathrm{e}$ $42^{\circ}$ dia de idade pela avaliação visual, considerando-se o número de vezes em que as aves inspiravam ar durante um minuto e com termômetro clínico veterinário com precisão de $0,1^{\circ} \mathrm{C}$, inserindose o termômetro a três centímetros na cloaca, respectivamente. No grupo TN, estes parâmetros foram avaliados entre $12 \mathrm{e} 13$ horas.

O delineamento foi feito em parcela subdividida "Split-Plot" no tempo. O efeito temperatura ambiente foi considerado parcela, enquanto que o efeito idade de avaliação de temperatura cloacal e freqüência respiratória, subparcelas. Os dados foram avaliados pela análise de variância, na qual foi testada a interação entre idades e grupos e posteriormente aplicou-se o teste de Tukey, a $5 \%$, conforme BANZATTO e KRONKA (2006).

\section{RESULTADOS}

Verificou-se interação significativa entre idade e grupos (ST e TN) para freqüência respiratória e temperatura cloacal.

Antes de serem submetidas à TACE, a 
Freqüência respiratória e temperatura cloacal em frangos de corte submetidos à temperatura ambiente cíclica elevada

menor média de MR/m no grupo ST foi observada no $42^{\circ}$ dia de idade, diferindo do $7^{\circ}, 14^{\circ}, 21^{\circ}$ e $28^{\circ}$ dias de idade. Já a maior média foi observada no sétimo dia, diferindo do primeiro, $28^{\circ}, 35^{\circ}$ e $42^{\circ}$ dias (Tabela 1). No TN, a menor ocorreu no $42^{\circ}$ dia e diferiu apenas do sétimo dia. Já a maior média foi obtida no sétimo dia e diferiu das demais idades.

Quando se compararam os grupos nas diferentes idades, antes das aves ST serem submetidas à TACE, houve diferenças no $1^{\circ}, 14^{\circ}, 21^{\circ}$ e $28^{\circ}$ dias com maiores médias de MR/m no TN, exceto no $1^{\circ}$ dia.

Depois que as aves ST foram submetidas à TACE (Tabela 2), este apresentou maior média de $\mathrm{MR} / \mathrm{m}$ no $35^{\circ}$ dia, que não diferiu de $28^{\circ}$ e $42^{\circ}$, contudo diferiu das demais; e a menor média foi obtida no primeiro dia de idade, diferindo das demais. Já o grupo TN apresentou a maior média no sétimo dia e esta diferiu das demais idades, a menor tendo sido obtida no $42^{\circ}$ dia, diferindo apenas do $7^{\circ}$ dia de idade.

TABELA1 - MÉDIAS E DESVIOS PADRÃO DOS MOVIMENTOS RESPIRATÓRIOS POR MINUTO (MR/M) DE FRANGOS DE CORTE MACHOS, ANTES DE SEREM EXPOSTOS À TEMPERATURA AMBIENTE CÍCLICAELEVADA (ST), POR UMA HORA DIÁRIAE NÃO EXPOSTOS (TN), DE JULHO AAGOSTO DE 2004, EM FRANCA-SP.

\begin{tabular}{cll}
\hline \multicolumn{3}{c}{ GRUPOS } \\
\hline Idade (dias) & \multicolumn{1}{c}{$\mathrm{ST}$} & \multicolumn{1}{c}{$\mathrm{TN}$} \\
\hline 1 & $47,2 \pm 4,5^{\mathrm{aCD}}$ & $48,4 \pm 4,8^{\mathrm{bBC}}$ \\
7 & $59,2 \pm 4,5^{\mathrm{aA}}$ & $56,8 \pm 9,9^{\mathrm{aA}}$ \\
14 & $58,2 \pm 3,9^{\mathrm{aAB}}$ & $52,4 \pm 4,1^{\mathrm{bBC}}$ \\
21 & $58,4 \pm 9,5^{\mathrm{aA}}$ & $46,8 \pm 5,9^{\mathrm{bBC}}$ \\
28 & $51,2 \pm 1,7^{\mathrm{aBC}}$ & $44,4 \pm 4,7^{\mathrm{bC}}$ \\
35 & $45,2 \pm 6,3^{\mathrm{aCD}}$ & $42,4 \pm 3,8^{\mathrm{aC}}$ \\
42 & $43,6 \pm 3,9^{\mathrm{aD}}$ & $42,0 \pm 4,7^{\mathrm{aC}}$
\end{tabular}

Médias com letras diferentes (maiúsculas na coluna e minúsculas na linha) indicam diferenças estatísticas $(P<0,05)$ pelo teste de Tukey.

TABELA2- $\quad$ MÉDIAS E DESVIOS PADRÃO DOS MOVIMENTOS RESPIRATÓRIOS POR MINUTO (MR/M) DE FRANGOS DE CORTE MACHOS, DEPOIS DAS AVES ST SEREM EXPOSTAS À TEMPERATURA AMBIENTE CÍCLICA ELEVADA (ST), POR UMA HORA DIÁRIA E NÃO EXPOSTOS (TN), DE JULHO A AGOSTO DE 2004, EM FRANCA-SP.

\begin{tabular}{ccl}
\hline & \multicolumn{2}{c}{ GRUPOS } \\
\hline Idade (dias) & \multicolumn{1}{c}{$\mathrm{ST}$} & \multicolumn{1}{c}{$\mathrm{TN}$} \\
\hline 1 & $73,2 \pm 17,5^{\mathrm{aC}}$ & $48,4 \pm 4,8^{\mathrm{bBC}}$ \\
7 & $132,8 \pm 27,7^{\mathrm{aB}}$ & $56,8 \pm 9,9^{\mathrm{bA}}$ \\
14 & $118,3 \pm 26,6^{\mathrm{aB}}$ & $52,4 \pm 4,1^{\mathrm{bBC}}$ \\
21 & $116,8 \pm 15,6^{\mathrm{aB}}$ & $46,8 \pm 5,9^{\mathrm{bBC}}$ \\
28 & $153,2 \pm 15,3^{\mathrm{aA}}$ & $44,4 \pm 4,7^{\mathrm{bC}}$ \\
35 & $160,4 \pm 10,7^{\mathrm{aA}}$ & $42,4 \pm 3,8^{\mathrm{bC}}$ \\
42 & $153,2 \pm 12,1^{\mathrm{aA}}$ & $42,0 \pm 4,7^{\mathrm{bC}}$
\end{tabular}

Médias com letras diferentes (maiúsculas na coluna e minúsculas na linha) indicam diferenças estatísticas $(P<0,05)$ pelo teste de Tukey 
MARCHINI, C.F.P. et al.

Ao comparar os grupos nas diferentes idades, depois que as aves ST foram submetidas à TACE, observou-se que este grupo apresentou maiores médias de MR/m do que TN em todas as idades.

A média de TC das aves ST, antes de serem submetidas à TACE (Tabela 3 ), foi menor no primeiro dia e diferiu das demais idades. Já as maiores ocorreram no $35^{\circ}$ e $42^{\circ}$ dias e não diferiu de $21^{\circ} \mathrm{e}$ $28^{\circ}$, todavia diferiu do $1^{\circ}, 7^{\circ}$ e $14^{\circ}$ dias. No TN, a menor média foi observada no primeiro dia e diferiu das demais idades. A maior média foi obtida aos 28 dias, mas não diferiu de 35 e 42 dias, entretanto diferiu de $1^{\circ}, 7^{\circ}, 14^{\circ}$ e $21^{\circ}$ dias. Assim, as aves de ambos os grupos tiveram aumento nas médias de TC com o aumento da idade.

Ao se comparar os grupos nas diferentes idades, antes das aves ST serem submetidas à temperatura ambiente elevada (Tabela 3 ), houve diferença no $14^{\circ} \mathrm{e} 28^{\circ}$ dias.

As aves ST tiveram a menor média de TC, depois de serem submetidas à TACE, no primeiro dia de idade, que não diferiu apenas do $7^{\circ}$ dia e a maior no $35^{\circ}$ dia, que não diferiu do $42^{\circ}$ dia, mas diferiu das demais (Tabela 4). Já o grupo TN apresentou a menor média no primeiro dia de idade e diferiu das demais. No $28^{\circ}$ dia observou-se a maior média e não diferiu de $35^{\circ}$ e $42^{\circ}$ dias, porém diferiu do primeiro, $7^{\circ}, 14^{\circ}$ e $21^{\circ}$ dia.

Quando se compararam os grupos nas diferentes idades, após a temperatura ambiente ser elevada, o grupo ST (Tabela 4) apresentou maiores médias de TC do que o TN, em todas as idades.

TABELA3- $\quad$ MÉDIAS E DESVIOS PADRÃO DAS TEMPERATURAS CLOACAIS (TC), EM ${ }^{\circ} \mathrm{C}, \mathrm{DE}$ FRANGOS DE CORTE MACHOS, ANTES DE SEREM EXPOSTOS À TEMPERATURAAMBIENTE CÍCLICA ELEVADA (ST), POR UMA HORADIÁRIAE NÃO SUBMETIDOS (TN), DE JULHO A AGOSTO DE 2004, FRANCA-SP.

\begin{tabular}{ccc}
\hline \multicolumn{3}{c}{ GRUPOS } \\
\hline Idade (dias) & \multicolumn{3}{c}{$\mathrm{ST}$} \\
\hline 1 & $39,9 \pm 0,3^{\mathrm{a}}$ & $40,0 \pm 0,1^{\mathrm{ab}}$ \\
7 & $40,7 \pm 0,3^{\mathrm{aC}}$ & $40,6 \pm 0,1^{\mathrm{aC}}$ \\
14 & $40,9 \pm 0,1^{\mathrm{aBC}}$ & $40,7 \pm 0,1^{\mathrm{bC}}$ \\
21 & $41,1 \pm 0,1^{\mathrm{aB}}$ & $41,0 \pm 0,1^{\mathrm{aB}}$ \\
28 & $41,1 \pm 0,1^{\mathrm{bAB}}$ & $41,4 \pm 0,1^{\mathrm{aA}}$ \\
35 & $41,2 \pm 0,1^{\mathrm{A}}$ & $41,2 \pm 0,1^{\mathrm{aAB}}$ \\
42 & $41,2 \pm 0,2^{\mathrm{aA}}$ & $41,2 \pm 0,2^{\mathrm{aA}}$
\end{tabular}

Médias com letras diferentes (maiúsculas na coluna e minúscula na linha) indicam diferenças estatísticas $(P<0,05)$ pelo teste de Tukey.

TABELA4- MÉDIAS E DESVIOS PADRÃO DAS TEMPERATURAS CLOACAIS $(T C)\left({ }^{\circ} \mathrm{C}\right)$ DE FRANGOS DE CORTE MACHOS, DEPOIS DE SEREM EXPOSTOS À TEMPERATURA AMBIENTE CÍCLICA ELEVADA (ST), POR UMAHORADIÁRIAE NÃO SUBMETIDOS (TN), DE JULHO A AGOSTO DE 2004, EM FRANCA-SP.

\begin{tabular}{ccc}
\hline & \multicolumn{2}{c}{ GRUPOS } \\
\hline Idade (dias) & ST & TN \\
\hline 1 & $40,9 \pm 0,3^{\mathrm{aE}}$ & $40,0 \pm 0,1^{\mathrm{bD}}$ \\
7 & $41,2 \pm 0,2^{\mathrm{aDE}}$ & $40,6 \pm 0,1^{\mathrm{bC}}$ \\
14 & $41,4 \pm 0,2^{\mathrm{aD}}$ & $40,7 \pm 0,1^{\mathrm{bC}}$ \\
21 & $41,8 \pm 0,3^{\mathrm{aC}}$ & $41,0 \pm 0,1^{\mathrm{bB}}$ \\
28 & $42,2 \pm 0,2^{\mathrm{a}^{\mathrm{B}}}$ & $41,4 \pm 0,1^{\mathrm{bA}}$ \\
35 & $42,9 \pm 0,6^{\mathrm{aA}}$ & $41,2 \pm 0,1^{\mathrm{bAB}}$ \\
42 & $42,7 \pm 0,4^{\mathrm{aA}}$ & $41,2 \pm 0,2^{\mathrm{bA}}$ \\
\hline
\end{tabular}

Médias com letras diferentes (maiúsculas na coluna e minúscula na linha) indicam diferenças estatísticas $(P<0,05)$ pelo teste de Tukey. 
Freqüência respiratória e temperatura cloacal em frangos de corte submetidos à temperatura ambiente cíclica elevada

\section{DISCUSSÃO}

O aumento da freqüência respiratória após as aves serem submetidas à temperatura ambiente elevada concorda com SILVA et al. (2003) que relataram aumento desta em aves de crescimento rápido quando submetidas ao estresse pelo calor. Esse resultado era esperado, uma vez que aves sob temperatura ambiente elevada aumentam a perda de calor por evaporação na tentativa de manter a homeotermia.

O aumento da freqüência respiratória nas aves é o mecanismo termorregulatório mais eficiente em temperatura ambiente elevada (OLIVEIRA NETO et al., 2000). Quando há aumento da temperatura do ar acima do conforto térmico, a dissipação de calor por radiação, condução e conveç̧ão diminui e a evaporação torna-se o principal processo de dissipação de calor (TEETER, 1994).

As médias de MR/m obtidos no TN mostram que em situações de termoneutralidade as aves não precisam utilizar intensamente os mecanismos como a ofegação para manter a temperatura corporal constante. EBERHART e WASHBURN (1993) consideram que aves com maiores índices de freqüência respiratória são menos resistentes ao calor.

O aumento da TC do ST observado depois do estresse pelo calor sugere que os mecanismos de dissipação de calor dessas aves foram insuficientes para atingirem a temperatura corporal observada no TN. Além disso, as aves do ST tiveram o seu "set-point" elevado, resultados que estão em acordo com os observados por TEETER et al. (1992).

A TACE tem considerável influência na temperatura corporal das aves, e o aumento observado neste parâmetro fisiológico pode ser um indicativo de que houve uma menor tolerância ao calor pelos frangos, com o aumento da idade. Segundo EBERHART e WASHBURN (1993), o aumento expressivo da temperatura corporal das aves sob estresse agudo pelo calor estão usualmente associadas a uma menor resistência ao mesmo e pode ser explicada pela redução na dissipação de calor para o ambiente, que já apresenta temperaturas elevadas (YALÇIN et al., 1997). Há que se considerar também que o frango de corte é suscetível ao estresse por calor porque a produção de calor metabólico aumenta com a idade, enquanto que a capacidade de dissipação, não
(TEETER, 1994). Assim, a retenção de calor metabólico afeta o crescimento das aves (DONKOH, 1989).

Na ave, uma conseqüência do aumento da TC em função de um acréscimo da temperatura ambiental é a redução do consumo de ração para evitar mais aporte de energia ao seu organismo (SEVEGNANI et al., 2005). O aumento na temperatura retal era esperado, pois, em ambientes com temperaturas altas, a perda de calor sensível, que depende da diferença entre a temperatura ambiente e a temperatura da pele, pode ser nula.

$\mathrm{O}$ aumento de TC com a idade, observado no ST e no TN está em acordo com os observados por SILVA et al. (2003) em aves de crescimento rápido.

\section{CONCLUSÃO}

Conclui-se que a temperatura corporal aumenta com a idade do frango, independentemente da temperatura ambiente, e a freqüência respiratória reduz com a idade para as aves em termoneutralidade. Frangos de corte submetidos à temperatura ambiente cíclica elevada apresentam aumento na freqüência respiratória e na temperatura cloacal.

\section{REFERÊNCIAS}

ALTAN, O.; PABUÇCUOGLU, A.; ALTAN, A.; KONYALIOGLU, S.; BAYRAKTAR, H. Effect of heat stress on oxidative stress, lipid peroxidation and some stress parameters in broilers. British Poultry Science, v. 44, n. 4, p. 545-550, 2003.

BANZATTO, D. A.; KRONKA, S. N. Experimentação agrícola. Jaboticabal: FUNEP, 2006, 237p.

BORGES, S. A.; MAIORKA, A.; SILVA, A. V. F. Fisiologia do estresse calórico e a utilização de eletrólitos em frangos de corte. Ciência Rural, v. 33, n. 5, p. 975-981, 2003.

DIONELLO, N. J. L.; MACARI, M.; FERRO, J. A.; RUTZ, F.; FERRO, M. I. T.; FURLAN, L. R. Respostas fisiológicas associadas à termortolerância em pintos de corte de duas linhagens por exposição a altas temperaturas. Revista Brasileira de Zootecnia, v. 31, n. 1, p.79-85, 2002. 
DONKOH, A. Ambient temperature: a factor affecting performance and physiological response of broiler chickens. International Journal of Biometeorology, v.33, n. 4, p. 259265, 1989.

EBERHART, D. E.; WASHBURN, K. W. Variation in body temperature response of naked neck and normally feathered chickens to heat stress. Poultry Science, v. 72, n. 8, p. 13851390, 1993.

FURLAN, R.L.; CARVALHO, N. C.; MALHEIROS, E. B.; MACARI, M. Efeito da restrição alimentar inicial e da temperatura ambiente sobre o desenvolvimento de vísceras e ganho compensatório em frangos de corte. Arquivo Brasileiro de Medicina Veterinária e Zootecnia, v. 53, n. 4, p. 1-7, 2001.

FURLAN, R. L.; MACARI, M. Termorregulação. In: MACARI, M.; FURLAN, R. L.; GONZALES, E. (Ed.). Fisiologia Aviária aplicada a frangos de corte. 2. ed. Jaboticabal: FUNEP/UNESP, 2002. p. 209-230.

OLIVEIRANETO, A. R.; OLIVEIRA, R. F. M.; DONZELE, J. L.; ROSTAGNO, H. S.; FERREIRA, R. A.; MAXIMIANO, H. C.; GASPARINO, E. Efeito da temperatura ambiente sobre o desempenho e características de carcaça de frangos de corte alimentados com dieta controlada e dois níveis de energia metabolizável. Revista Brasileira de Zootecnia, v. 29, n. 1, p. 183-190, 2000.
SEVEGNANI, K. B.; CARO, I. W.; PADORFI, H.; SILVA, I. J. O.; MOURA, D. J. Zootecnia de precisão: análise de imagens no estudo do comportamento de frangos de corte em estresse térmico. Revista Brasileira de Engenharia Agrícola e Ambiental, v. 9, n. 1, p. 115-119, 2005

SILVA, M. A. N.; HELLMEISTER FILHO, P.; ROSÁRIO, M. F.; COELHO, A. A. D.; SAVINO, V. J. M.; GARCIA, A. A. F.; SILVA, I. J. O.; MENTEN, J. F. M. Influência do sistema de criação sobre o desempenho, condição fisiológica e o comportamento de linhagens de frango de corte. Revista Brasileira de Zootecnia, v. 32, n. 1, p. 208-213, 2003.

TEETER, R. G. Optimizing production of heat stressed broilers. Poultry Digest, v. 53, p. 10-27, 1994.

TEETER, R. G.; SMITH, M. O.; WIERNUSZ, C. J. Research Note: Broiler acclimation to heat distress and feed intake effects on body temperature in birds exposed to thermoneutral and high ambient temperatures. Poultry Science, v. 71, n. 6, p. 1101-1104, 1992.

YALÇIN, S.; SETTAR, P.; OZKAN, S.; CAHANER, A. Comparative evaluation of three commercial broiler stocks in hot versus temperate climates. Poultry Science, v. 76, n. 7, p. 921-929, 1997. 